\title{
Immune Deposits in Glomerular Diseases and Their Clinical, Histopathological and Immunopathological Correlation
}

\author{
Md. Towhid Hossain 1 , Morshida Begum², \\ AJE Nahar Rahman ${ }^{3}$, Mohammed Kamal ${ }^{4}$
}

\begin{abstract}
Glomerulonephritis (GN) is a common renal disease and common cause of chronic renal failure (CRF) accounts for more than one-third of patients of end stage renal disease (ESRD) requiring either dialysis or transplantation. In our country, early diagnosis and treatment of GN depends on routine urine and blood examination and using light and immunofluorescent microscopic study of renal biopsy. The purpose of this study was to demonstrate the frequency, type, intensity, pattern and site of deposition of immunoglobulin $\lg G, \operatorname{lgA}$, IgM and C3 by direct immunofluorescence microscopic technique (DIF) in various pattern of $G N$ and to correlate with clinical and histopathological findings.

Among 120 cases of renal biopsy, 110 cases (91.67\%; $n=120$ ) were adequate for histopathologic study only and 98 cases $(81.67 \% ; n=120)$ were adequate for both histopathologic and direct immunofluorescence microscopic study. In this series, maximum numbers of cases were found in 21-30 age group (27.27\%). Most frequent clinical presentation and pattern of glomerulonephritis were nephrotic syndrome $(61.22 \% ; n=98)$ and mesangioproliferative GN (40.81\%) respectively. Among 98 cases of study group, 49 cases (50\%; $n=98)$ were DIF positive. The most frequent type of depositions were C3 (type) in various combinations (98\%; $n=49)$ followed by $\operatorname{lgG}(67.35 \%)$ and $\operatorname{lgA}$ $(40 \%)$. Mesangium followed by glomerular basement membrane were the most frequent site and granular pattern was the most frequent pattern of deposition. The frequent combination of depositions in various pattern of $G N$ were C3 + IgG (36.73\%; $n=49)$ followed by C3 + IgA (20.41\%). There was a correlation between histopathologic pattern of GN and type-site-pattern of deposition in the glomeruli. Immune-depositions were cent percent in IgA nephropathy, membranous GN (MGN), diffuse proliferative GN and membranoproliferative GN. Among 15 cases of $\operatorname{lgA}$ nephropathy (15.31\%; $n=98)$, most frequent pattern and clinical presentation of $G N$ was mesangioproliferative GN $(60 \% ; n=15)$ and haematuria $(46.67 ; n=15)$ respectively.

In this study, DIF was proved to be essential, sensitive and specific diagnostic tool in the evaluation of glomerular diseases. However, DIF study is no substitute of light microscopy but both provide information which when taken as a whole contributes to better understanding of $G N$.
\end{abstract}

Key words : DIF, GN, CRF, ESRD

\section{Introduction}

Glomerulonephritis is the common renal disease and also common cause of chronic renal failure in both developed and developing countries ${ }^{1}$. Renal biopsy remains the "gold standard" for the diagnosis of glomerular diseases. To evaluate kidney biopsy, one should correlate complete clinical and laboratory information with light, immunofluorescence and electron microscopic findings. Most of the glomerulopathies are still classified and named according to their morphologic features. A given clinical syndrome can be associated with several histopathologic patterns. Focal as well as diffuse proliferative $G N$, crescentic and mesangial $G N$ usually present with nephritic syndrome. Classic pathologic correlate of the nephritic syndrome is proliferative $\mathrm{GN}^{2}$. The morphologic pattern affecting the glomerular filtration barrier for protein, namely the glomerular basement membrane (GBM) and visceral epithelial cells are membranous $G N$, minimal change disease (MCD) and focal segmental glomerulosclerosis (FSGS)-typically present with nephrotic range proteinuria ${ }^{2}$. Membranoproliferative $G N$ is a hybrid lesion that presents a combination of nephritic-nephrotic features. Glomerular deposition diseases can also trigger nephrotic type, nephritic type response or combination of both and thus show marked clinical and morphologic overlap ${ }^{2}$. The site of the antibody deposition within the glomerulus is a critical determinant of clinico-pathologic presentation.

1. Dr. Md. Towhid Hossain, Assistant Professor, Department of Histopathology, National Institute of Kidney Diseases and Urology (NIKDU), Dhaka

2. Dr. Morshida Begum, Assistant Professor, Department of Radiology and Imaging, Bangabandhu Sheikh Mujib Medical University ( $B S M M U)$, Dhaka.

3. Dr. A J E Nahar Rahman, Chairman, Department of Pathology, Bangabandhu Sheikh Mujib Medical University (BSMMU), Dhaka.

4. Dr. Mohammed Kamal Professor, Department of Pathology, Bangabandhu Sheikh Mujib Medical University BSMMU, Dhaka. 
Relatively anionic antigens are repelled by the GBM and trapped in the sub-endothelial and mesangial location usually causing proliferative GN2. Relatively cationic antigens tend to permeate GBM and deposits within sub-epithelial aspects of GBM and visceral epithelial cells causing nephrotic type response. Acute sub-endothelial deposits are responsible for leukocyte infiltration, vasoconstriction and thrombotic microangiopathy causing nephritic syndrome. But sub-epithelial, intramembranous immune-deposits usually cause nephrotic range proteinuria ${ }^{2}$.

Direct immunofluorescence microscopic (DIF) study has resulted in a considerable expansion in the understanding of human glomerular diseases ${ }^{3}$. It seems that there is a need to further study of the pattern of GN after applying the usual histopathologic technique. By DIF technique, it can correlate sometimes significantly with light microscopic diagnosis and clinical syndromes but impossible to group patients into any specific categories3. Immune-deposits of $\operatorname{lgG}-\operatorname{lgA} / \mathrm{C} 3$ were found higher frequency in patients of haematuria due to secondary cause and patients with immunedeposits of IgAVC3 had recurring respiratory infection4. Since the treatment of glomerulonephritis is often determined by the histopathologic findings, any technique that identifies the particular lesion quickly, easily and definitely may be of clinical assistance. It is the combination of great sensitivity and specificity together with the use of histologic technique that makes immunofluorescence technique so useful. Therefore, an attempt to correlate the findings of this technique with the clinical and histological diagnosis will further enhance its reliability.

In the light of above context, renal biopsies were analyzed to detect and correlate the findings of DIF with the various pattern of glomerulonephritis and clinical syndromes.

\section{Material and Methods}

Renal biopsy (needle biopsy) specimens were obtained from the clinically suspected and biopsy indicated patients of glomerulonephritis (GN) after proper willen and vecbul consent and studied in the Department of Pathology, Bangabandhu Sheikh Mujib Medical University (BSMMU), Dhaka and in the Histopathology Department of Armed Forces Institute of Pathology (AFIP), Dhaka during the period from January/1999-December/1999.

Patients were selected from the Department of Nephrology, Bangabandhu Sheikh Mujib Medical
University (BSMMU), Dhaka, Dhaka Medical College Hospital, Combined Military Hospital (CMH) and few other referral hospitals of Dhaka city.

One hundred and twenty (120) clinically diagnosed cases of nephrotic syndrome, nephritic syndrome, asymptomatic hematuria or proteinuria etc of various age groups were biopsied and preliminary included in this study. Clinical information was recorded in a pre-designed proforma before doing histopathology and DIF study. Renal biopsies were performed by Nephrologist. At least two specimens of $12-20 \mathrm{~mm}$ in length renal tissues, one in $10 \%$ formalin for histopathology (light microscopic study) and another one in normal saline for DIF study were taken. Cryostat machine (for frozen section), fluorescent microscope (NIKON; Labophot-2, model-661012, Japan), micropipette, phosphate buffer saline (PBS) and commercially prepared fluorescein isothiocyanate (FITC) conjugated goat antihuman $\lg G, \lg A, \lg M$, and C3 reagent (Sanofi Diagnostics Pasteur, Inc. 1000 lake Hazeltine Drive, Chaska, USA, MN 55318. Cat-800-666-5111) were used for DIF study. Formalin-fixed processed tissue section were stained by both haematoxylin-eosin ( $H$ \& E) and Periodic Acid Schiff (PAS) stain for histopathologic study. GBM thickness was observed under light microscope with PAS stain.

Histopathology of the biopsied specimens were performed by the author himself and thoroughly verified by at least two Histopatholgists-the Head of the Department of Pathology and the Professor of Pathology, BSMMU, Dhaka. The DIF study was performed by the Head of the Department of Histopathology of Armed Forces Institute of Pathology (AFIP), Dhaka and the author himself along with verification by the Head of the Department of Pathology, BSMMU, Dhaka.

Following were the criteria to define different terms and various patterns of $G N$.

Adequate for DIF study: Presence of at least one glomerulus under fluorescent microscope. Adequate for light microscopic study: Presence of at least 5 glomeruli under light microscope ${ }^{5}$. Mesangial proliferation: More than 3 mesangial cells embedded in matrix of one segment. Endothelial proliferation: More than 2 nuclei per capillary loops and leukocyte infiltration: More than 5 leukocytes per glomerulus6. Crescentic GN: When at least $50 \%$ of the glomeruli involved as crescents and minimal change disease (MCD) when no evidence of any change or presence of minimal mesangial proliferation of less than $50 \%$ of the glomeruli in a section 7 . Lesions are classified as focal or diffuse proliferative when they 
involve the minority $(<50 \%$ ) or majority $(250 \%)$ of glomeruli respectively2. IgA nephropathy: Predominant mesangial deposition of $\operatorname{lgA}$ along with C3 detected by immunofluorescence microscopy8. Clinical presentation and patterns of 15 cases of $\operatorname{lgA}$ nephropathy in this study were evaluated separately. Focal segmental glomerulosclerosis (FSGS): Segmental (one or two lobules) sclerosis with hyalinosis involving portions of fewer than $50 \%$ of the glomeruli in a section. Sclerosis was defined as increase in amount of homogeneous non-fibrillar extracellular material of similar composition to GBM and mesangium.

Membranous GN: Diffuse thickening of GBM due to sub-epithelial deposits of immune complex without evidence of inflammation or cellular proliferation. Membranoproliferative GN: Diffuse thickening of GBM with predominant proliferation of mesangial cells and extension of matrix often with interposition in between the endothelial cells and GBM causing tram-track appearance under light microscope 2 . Mesangioproliferative GN: Diffuse increase in glomerular cellularity predominantly due to mesangial cells often with concomitant increase in mesangial matrix 9 .

Photographs were taken in both cases of light microscopic and DIF study.

Fluorescein dye conjugated antihuman antibodies (IgG, $\lg A, \lg M$ and $C 3$ ) were fixed with the tissue section by ten times diluted anti-sera. Then fall of ultraviolet light into the stained tissue section become excited and emit light of higher wave length to be visible (apple green colour) under fluorescent microscope if there is any antigen in the tissue section. Here antibodies and complement lies within the tissue section (if any) act as antigen and artificially prepared antihuman antibodies and complement act as antibody.

Total number of glomeruli, GBM, mesangial, endothelial and epithelial cells and infiltration of inflammatory infiltrates, tubules, interstitium and blood vessels were observed under light microscope 6,7 .

Type (IgG, IgA, IgM and C3), pattern (granular or linear), site (GBM or mesangium) and intensity of deposition in the glomeruli were observed under fluorescent microscope. The intensity of FITC staining was graded subjectively from 0 to +3 : 0 being negative and +3 maximum intensity (mild +1 ; moderate ++2 ; marked +++3$)^{6,7}$.

The clinical information were recorded, then histopathological diagnosis of formalin-fixed processed renal tissue were made based on H\&E and PAS stain followed by saline-fixed tissue frozen section under -25 degree centigrade temperature, DIF staining and immunofluorescence microscopic study. These three findings were then correlated and evaluated.

\section{Results}

Out of 120 renal biopsies, 10 cases were labeled as inadequate for histopathologic study and 12 cases showed no glomeruli and inadequate for DIF study. Clinical, histopathological and DIF study were done among remaining 98 cases. Among 98 study cases, 56 were male $(57 \%)$ and $42(43 \%)$ were female. The age ranged from 8-70 years and mean age of 30.67 years. Mesangioproliferative GN $(40.81 \% ; n=98)$ was the most frequent pattern of $\mathrm{GN}$ followed by focal segmental proliferative GN $(20.41 \%)$, IgA nephropathy $(15.31 \%), \operatorname{MCD}(10.21 \%)$ and membranoproliferative GN $(10.21 \%)$ of all age group. The most frequent clinical presentation of the study group was the nephrotic syndrome $(61.22 \%)$ followed by haematuria $(11.22 \%)$ and nephritic syndrome $(11.22 \%)$ (Table-1). Among fifteen cases (15.31\%; $n=98$ ) of IgA nephropathy, there were $60 \%$ mesangioproliferative GN, $26.75 \%$ CGN, $6.7 \%$ MPGN and $6.75 \%$ focal segmental proliferative GN of histopathologic pattern in this study group. Sixty cases $(61.22 \%$; $n=98)$ presented with nephrotic syndrome showed as pattern of mesangioproliferative GN (25.51\%), focal segmental proliferative GN $(11.22 \%)$, MCD $(10.21 \%)$, MPGN $(6.13 \%)$, membranous GN $(4.08 \%)$, crescentic GN $(2.04 \%)$ and lupus nephritis $(2.04 \%)$. On the other hand, $40(40.81 \%)$ cases of mesangioproliferative GN of this study clinically presented with nephrotic syndrome $(25.51 \%)$, haematuria $(7.15 \%)$, nephritic syndrome $(1.02 \%)$, CRF $(1.02 \%)$, proteinuria $(5.10 \%)$ and RPGN $(1.02 \%)$. Twenty $(20.41 \%)$ cases of focal segmental proliferative $G N$ were clinically presented with nephrotic syndrome $(\mathbf{1 1 . 2 1 \% )}$ ), haematuria $(3.06 \%)$, nephritic syndrome $(4.08 \%)$ and proteinuria $(2.04 \%$ ) (Table-1). Hundred percent of MCD, membranous $G N$ and lupus nephritis, $66.67 \%$ of crescentic $\mathrm{GN} \quad(n=3), 62.51 \%$ of mesangioproliferative GN $(n=40), 55 \%$ of focal segmental proliferative $\mathrm{GN}(n=20)$ and $60 \%$ of membranoproliferative $\mathrm{GN}(n=10)$ were presented with nephrotic syndrome. Sixty-seven percent (66.67\%; n=3) of FSGS, $20 \%$ of focal segmental proliferative $\mathrm{GN}(n=20), 100 \%$ of diffuse proliferative $\mathrm{GN}(n=2)$ and $30 \%$ of membranoproliferative $\mathrm{GN}$ $(n=10)$ were presented with nephritic syndrome.

Forty-seven percent of IgA nephropathy ( $n=15)$, 
$17.5 \%$ of mesangioproliferative $\mathrm{GN}(n=40)$, and $10 \%$ of membranoproliferative $\mathrm{GN}(n=10)$ were presented with haematuria. Among 110 histopathologically adequate cases, 12 cases were left out from DIF study because of 'no glomeruli' under fluorescent microscope. DIF study was done in the rest 98 cases of which 49 cases (50\%) were DIF positive and another 49 cases $(50 \%)$ DIF negative (Table11). The sites of deposition were predominantly in the mesangium in case of IgA nephropathy, mesangioproliferative GN, FSGS and focal segmental proliferative $\mathrm{GN}$ and in the GBM in case of membranous and membranoproliferative GN. All of the glomerular depositions were granular in pattern. The most frequent type of single immunedeposits in various combination was C3 $(98 \%, n=$ $49)$ followed by $\lg$ ( $67.35 \%, 33$ out of 49 cases) and $\lg A(40 \%)$. The most frequent combination of deposits were $\lg \mathrm{G}+\mathrm{C} 3$ found in 18 cases $(36.73 \%$, $\mathrm{n}=49$ ) followed by $\lg A+C 3$ in $(20.41 \%)$ cases (Table111). Most frequent presentation in IgA nephropathy was haematuria $(46.7 \% ; n=15)$ followed by nephrotic syndrome $(26.7 \%)$. The most frequent pattern of GN in $\lg A$ nephropathy was mesangioproliferative $\mathrm{GN}$ $(60 \% ; n=15)$. In IgA nephropathy, depositions were mesangial, granular and mostly IgA along with mild deposition of C3 combination. DIF positivity of IgA nephropathy, MGN, MPGN and diffuse proliferative GN and lupus nephritis of the present study were cent percent but positivity of mesangioproliferative GN, MCD and crescentic GN were 36.67, 18.18 and 33.33 percent respectively (Table-1V).

\section{Table-1}

Correlation of clinical presentation and histopathologic pattem ( $n=98)$

\section{Clinical}

foatures

\begin{tabular}{|c|c|c|c|c|c|c|c|}
\hline Monderon & Fockid & WCD & IPCN & 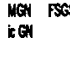 & Cumonts & $D P C H$ & cant \\
\hline
\end{tabular}

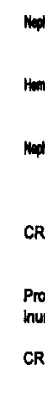

25

\begin{tabular}{|c|c|c|c|c|c|c|c|c|}
\hline 11 & 100 & $\begin{array}{l}06 \\
\text { (B.13) }\end{array}$ & $\begin{array}{l}14 \\
4.000\end{array}$ & $\infty$ & $\begin{array}{l}02 \\
(201)\end{array}$ & $\boldsymbol{\infty}$ & $\infty$ & 20191212 \\
\hline
\end{tabular}

07

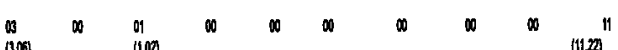

0101011

(1.02) (4.08) (3.06)

(2.04)

(1.02)

(11.22)

CRF

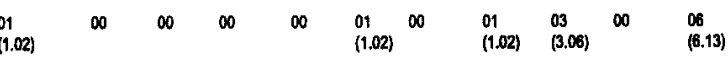

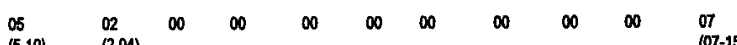

(5.10) (2.04) (07-15)

$\begin{array}{lllllllllll}04 & \infty & \infty & \infty & \infty & 00 & 01 & \infty & 01 & \infty & 03 \\ (1.02) & & & & & & (1.02) & & (1.02) & & (6.13)\end{array}$

$\begin{array}{llllllllllll}\text { RPGN } & 40 & 20 & 10 & 10 & 04 & 03 & 03 & 02 & 04 & 02 & 99 \\ & (40.81) & (20.41) & (10.21) & (10.21) & (40.08) & (3.06) & (3.06) & (2.04) & (4.08) & (2.04)) & (100\}\end{array}$
Table-II

Histopathologic pattern and DIF findings in 98 study cases

\begin{tabular}{lcclll}
\hline & Cases & DIF positive & DIF negative \\
\hline Mesangioproliferative GN & 30 & 11 & 36.67 & 19 & 63.33 \\
Focal segmental proliferative GN & 18 & 03 & 16.67 & 15 & 83.33 \\
IgA nephropathy & 15 & 15 & 100 & 00 & 000 \\
Minimal change disease & 11 & 02 & 18.18 & 09 & 81.82 \\
Membranoproliferative GN & 08 & 08 & 100 & 00 & 000 \\
Membranous GN & 04 & 04 & 100 & 00 & 00 \\
FSGS & 03 & 00 & 000 & 03 & 100 \\
Crescentic GN & 03 & 01 & 33.33 & 02 & 66.67 \\
Diffiuse protifeative GN & 02 & 02 & 100 & 00 & 000 \\
Chronic glomerulonephritis & 01 & 00 & 000 & 01 & 100 \\
Lupus nephritis & 02 & 02 & 100 & 00 & 00 \\
$\quad$ Total & 98 & 49 & 50 & 49 & 50
\end{tabular}

\section{Table-III}

Distribution of immunoglobulin $\lg G, \lg A, \lg M$ an $C 3$ of 49 DIF positive cases in varius pattem of $\mathrm{GN}$

\begin{tabular}{lcl}
\hline Immunoglobulin and $C 3$ & No. of cases $(n=49)$ & Percentage \\
\hline $\lg G+C_{3}$ & 18 & 36.73 \\
$\lg A+C_{3}$ & 10 & 20.41 \\
$\lg G+\lg M+C_{3}$ & 06 & 12.24 \\
$\lg A+\lg G+C_{3}$ & 05 & 10.20 \\
$\lg G+\lg A+\lg M+C_{3}$ & 04 & 08.16 \\
$\lg M+C_{3}$ & 03 & 6.12 \\
$\lg A+\lg M+C_{3}$ & 01 & 04.04 \\
$C 3$ alone & 01 & 04.04 \\
$\lg M$ alone & 01 & 04.04 \\
Total & 49 & 100
\end{tabular}

Table-1V

Comparative study of DIF positive cases by different authors

\begin{tabular}{|c|c|c|c|c|c|c|c|}
\hline \multicolumn{3}{|l|}{ Pattem of GN } & \multicolumn{5}{|c|}{ DIF positive findings in percentage } \\
\hline More & $\begin{array}{l}1972 \\
\text { 1-Merogor. }\end{array}$ & $\begin{array}{l}\text { Larsen, } \\
1978\end{array}$ & $\begin{array}{c}\text { Metha ot al, } \\
1983\end{array}$ & $\begin{array}{l}\text { Tebaseum, } \\
1988\end{array}$ & $\begin{array}{c}\text { Sharmin } \\
1994\end{array}$ & $\begin{array}{l}\text { Nabir, } \\
1996\end{array}$ & $\begin{array}{l}\text { Present } \\
\text { study }\end{array}$ \\
\hline $\begin{array}{l}\text { Mesanglopro } \\
\text { liferative }\end{array}$ & 53.33 & 85 & 45 & 80 & 70 & 60 & 36.67 \\
\hline Focal proliferative & 74.58 & 90.67 & 65 & 60 & 50 & 40 & 52.48 \\
\hline MCD & $\mathbf{0 0}$ & 60 & 57.1 & $\infty$ & $\infty$ & 00 & 18.18 \\
\hline Membranous GN & 100 & 100 & 100 & 100 & 100 & 100 & 100 \\
\hline MPGN & 100 & 100 & 100 & 100 & 100 & 100 & 100 \\
\hline IgA nephropathy & 100 & 100 & 100 & 100 & 100 & 100 & 100 \\
\hline Diffuse prolinerative & $\mathbf{8 3 . 8 8}$ & 77.7 & 100 & 100 & 100 & 100 & 100 \\
\hline $\operatorname{CGN}$ & 00 & $\mathbf{0 0}$ & $\mathbf{0 0}$ & 00 & 50 & 00 & $\infty$ \\
\hline Crescentic $\mathrm{GN}$ & 76 & 65 & 33 & 100 & 100 & 00 & 33.33 \\
\hline FSGS & 76.32 & 40 & 60 & 33.33 & 10 & 00 & 66.67 \\
\hline Lupus nephritis & 100 & 100 & 100 & 100 & 100 & 100 & 100 \\
\hline
\end{tabular}

\section{Discussion}

In the present study, the maximum number of cases occurred in the 21-30 age group followed by 11-20 and $31-40$ age groups. Nephrotic syndrome was the most frequent clinical presentation shown by Cameron, $1980(57.77 \%)^{100}$. Ziauddin et al, $1993(56.6 \%)^{11}$ and Rahman et al, $1984(94 \%)^{12}$ similar to our study $(61.22 \%)$. There was a wide variation regarding the commonest pattem of glomerulonephritis yet done in home and abroad. 
In the study of Rahman et al in 1984, Ziauddin et al in1993 and Nabir et al in 1996 showed that MCD, diffuse proliferative $\mathrm{GN}$ and focal segmental proliferative $\mathrm{GN}$ were the most frequent pattern of GN respectively ${ }^{13}$. But the study of Tabassum, 1988 and Sharmin, 1994 along with our study reveal mesangioproliferative $\mathrm{GN}$ is the most frequent pattern of $\mathrm{GN}^{14,15}$. Diffuse proliferative $\mathrm{GN}$ is the commonest pattern of $\mathrm{GN}$ in western world $16,17,18$. It needs serial sections and examination of more glomeruli during microscopic study to differentiate MCD from focal segmental proliferative GN. In fact, mesangial cellularity of MCD is an intermediate step in the evolution of MCD to FSGS ${ }^{19}$. By light microscopy, evidence of segmental proliferations may be missed if the biopsy specimen contains an insufficient number of glomeruli. Clinico-pathologic correlation of different patterns of $\mathrm{GN}$ in this study group was more or less similar to other studies ${ }^{2}$.

In this study, DIF was positive in 49 cases (50\%) and negative in another 49 cases $(50 \%)$. Similar results were obtained by other two investigators done in Bangladesh ${ }^{14,15}$. Our study also reveals predominantly generalized distribution of deposits either in the mesangium or GBM $(93.88 \%)$ rather than focal (6-12\%) which signifies usual generalized involvement of immune-deposits in glomerular diseases. More or less similar findings were reported by other authors $14,15,20,21$.

Conventionally, apparently normal glomeruli in light microscope and no deposition in DIF study regarded as MCD. But our study reveals DIF positivity of $18.18 \%$ in MCD cases similar with a few other studies $^{4}, 21$. Brenner and Rector in 2004 explained about DIF positivity due to differentiation between mild prominences of mesangial cells as observed in MCD and definite mesangial proliferation is subjective and highly susceptible to artifacts of sectioning and specimen preparation ${ }^{5}$.

The most frequent type of single immune-deposits in various combinations was IgG followed by $\mathrm{C} 3$ and IgA in the study of Tabassum, 1988 and Nabir, 1996dissimilar to our study (C3 followed by $\lg G$ and $\lg A$ ). Regarding most frequent presentation (haematuria), pattern and combination of deposition $(\lg A+C 3)$ of present study in IgA nephropathy also showed very similar result reported by Sharmin, 1994 and Khan et al, $1990^{15}, 23$. It was also observed that the type, site, pattern and intensity of immune-depositions greatly modify the clinical presentation and course of GN2.There is a variation of DIF positivity by different authors done in home and abroad $24,4,13,14,15$ (Table- 1 V).

\section{Conclusion}

Our study revealed that many of the glomerulonephritis were immune-deposit mediated causing different patterns of $\mathrm{GN}$ and clinical syndromes. IgA nephropathy showed mes angiopr oliferative,membranoproliferative, chronic glomerulo nephritis and focal segmental proliferative phenotypes of GN. In this study, most of the patterns of GN were diagnosed by light microscopy; a few of them such as IgA nephropathy-DIF study was essential. The extent of focal lesion might misjudge in a small biopsy specimen and sections through glomeruli might miss segmental lesions. It needs large samples as well as serial sections with more glomeruli to diagnose MCD, FSGS and focal segmental proliferative GN properly. Though somewhat expensive, DIF was probed to be simple, rapid, sensitive and highly specific diagnostic procedure. The site, type, pattern and intensity of immune-deposits was identified by fluorescent microscope but to identify the exact location of the site (sub-endothelial or sub-epithelial or intramembranous) of immune deposits, electron microscope is needed. It was observed that proper diagnosis and treatment of glomerular diseases depend on correlation of clinical, light and fluorescent as well as electron microscopic study.

We can conclude with an expectation of introducing immunofluorescence technique as an essential diagnostic tool for the evaluation of renal biopsy in Bangladesh.

\section{References}

1. Rahman $M$. Chronic renal failure. Bangladesh renal journal.1987; vol-6 (2): 39-42.

2. Hugh R Brady, Yvonne M, O'Meara, Barry M Brenner. Glomerular Diseases. Glassock RJ, Brenner BM. Harrison's Principles of Internal Medicine. 16th edition. In. New York. Mcgraw-Hill Company. 2005: 1674-1690.

3. Larsen S and Brun C. Immune deposits in human glomerulopathy Acta Pathol Microbiol Scand A. 1979 Sep; $87 A(5)$ : 321-33.

4. Larsen S. Immunofluorescence microscopic findings in minimal or no change disease and slight mesangioproliferative glomerulonephritis. Acta Path Micrbiol Scand. 1978; Sect A.86: 521-42.

5. Brenner and Rector's THE KIDNEY. 7th edition. Independence square west, Philadelphia. The cutris centre. 2004; Vol-1:1301-1310.

6. Rotter W. Colour Atlas of Kidney Biopsy. Alan R. Liss, Inc. New York. 1985;1-54. 
7. Rosai Jaun and Ackerman Lauren V. Ackerman's Surgical Pathology. 8th edition. Anne S Patterson. Mosby year book. Inc. 11830, Westline industrial drive, ST Luis. 1996; 1: 1064-1105.

8. Vinay kumar, Abul K Abbas, Nelson Fausto. Robbins and Cotran Pathologic Basis of Disease. 7th edition. The Cutris Centre, Philadelphia, Pensylvania, 19106. Saunders An imprint of Elsevier. 2004; 986.

9. Shaul G Massry, Richard J . Messry and Glassock's Text Book of nephrology. 4th edition. 530, Walnur street, Philadelphia, USA. Williams and Wilkins. $2001 ; 717$.

10. Cameron JS, Turner DR and Ogg CS. The nephrotic syndrome in adults with minimal change glomerular lesion. Q Med J. 1974; 43: 461-488.

11. Ziuddin A and Hossain A. Clinicopathologic analysis of glomerulonephritis. Bangladesh Armed Forces Medical Journal.1993; Vol-XV11: 51-54.

12. Rahman $T$, Islam $N$, Rashid $H U$ and Rahman $M$. Morphological spectrum of glomerular disease presentating with nephrotic syndrome- an experience based on light microscopy of needle biopsy. Bangladesh Renal Journal. 1984; 3 (2): 1-5.

13. Nabir Uddin. Pattern of glomerulonephritis in Bangladesh: Correlation of light microscopy and immunofluorescent study. FCPS histopathology dissertation. .Dhaka, IPGM\&R. 1996; 45-67.

14. Tabassum S, Rahman KM, Mamun KJ, Ahmed AN, Islam KM and Rahman $H$. Immunofluorescence microscopic findings in glomerulonephritis. Bangladesh Medical Research Council Bulletin.1998; 23(3):77-81.

15. Sharmin F. IgA nephropathy in teaching hospitals. M Phil thesis. University of Dhaka. 1994; 24-27.
16. Rashid HU, Morely AR and Merr DNS. Serum complement in mesangiocapillary GN. Bangladesh Renal Journal. 1981; 1 (2): 6-11.

17. Cheong I, Kong $N$ and Sagasothy $M$. IgA nephropathy in Malaysia. South Asian J Top Med Public Health. 1991; 22: 120-122.

18. Alfanzo JP, Landells JW and Daniel $S$. Glomerulonephritis: A preliminary clinico-pathological reports on 29 cases. Ethiopian Med J. 1982; 20: 2732.

19. John Feehally, Jorgen Floge, Richard J Johnson. Comprehensive Clinical Nephrology. 3rd edition. John F Kennedy Blvd, Ste-1800. Mosby Elsevier. 2007; 211-212.

20. Larsen $\mathrm{S}$ and Brun C. Immune deposits in human glomerulopathy Acta Pathol Microbiol Scand A. 1979 Sep; 87A (5): 321-33.

21. Metha RI, Ganguly NK, Sehal PC and Chugh KS. Circulating immune-complexes in glomerulonephritis with special reference to minimal change disease. 1983; 77: 96-106.

22. Chen YP, Wang $H Y$ and Zon WZ. Non IgA Mesangioproliferative GN. Clinical and Pathological analysis of 77 cases. Chin Med J. 1994; 102: 510515.

23. Khan TN, Sinniah $R$ and Nagvi AG. IgA nephropathy in Pakistan. Journal of Pakistan Medical Association. 1990; 31-36.

24. Moral-Marogor L, Leathem $A$ and Richet $G$. Glomerular abnormalities in non-systemic diseases. Relationship between findings of light microscopy and immunofluorescence in 433 renal biopsy specimens. Am J Med. 1972; 53:170-84. 